\title{
Adapted tool design for the cold forging of gears from non-ferrous and light metals
}

\author{
Andreas Rohrmoser $^{1}$ (D) Hinnerk Hagenah ${ }^{1} \cdot$ Marion Merklein $^{1}$ \\ Received: 29 September 2020 / Accepted: 1 December 2020 / Published online: 23 January 2021 \\ (C) The Author(s) 2021
}

\begin{abstract}
Due to growing competitive pressure within the manufacturing sector, there have been increasing attempts to establish resource saving production methods in gear manufacturing within recent years. Cold forging offers the potential—in addition to a high material and energy efficiency - to produce gears with an excellent surface quality, increased hardness as well as a load adapted fiber orientation. With regard to the wide range of applications there is a broad demand for gear materials, ranging from highstrength steels to non-ferrous and light metals. The flow behavior of the material has a significant influence on the cold forging process. Therefore, no consistent process result is achieved when forming different materials. Challenges exist due to deficient die filling and poor resulting geometrical accuracy. In this contribution, material-specific challenges during the full forward extrusion of gears from non-ferrous and light metals have been identified and suitable tool-sided measures were derived. A validated numerical process model was used to determine the underlying mechanisms of action and to verify the derived measures. A reduced yield stress leads to inflow formation, insufficient die filling, and low achievable strain hardening, as well as gearing accuracy. The tool-sided measures achieved a significant increase of resulting die filling and gearing accuracy as well as the mechanical properties. That provides the basis for the production of ready-to-use gears from various metal materials.
\end{abstract}

Keywords Cold forging $\cdot$ Gears $\cdot$ Light metals $\cdot$ Tool design $\cdot$ Simulation

\section{Introduction}

Gears represent integral and essential machine elements within drive technology and are applied in large quantities in mechanical engineering and the automotive industry. Also, in the future, a sustained growth of the market for gears, drives, and speed changers is expected [1]. Conventionally, the manufacturing of gears is done by machining processes due to their flexibility and low investment costs [2]. Against the background of high production volumes, forming processes such as full forward extrusion offer potential with regard to productivity [3] and resource saving use of material [4]. The application, however, is limited due to the low achievable die filling and gearing accuracy [2]. In previous research, influencing parameters on the material flow such as the

Andreas Rohrmoser

andreas.rohrmoser@fau.de

1 Institute of Manufacturing Technology, Department Mechanical Engineering, Friedrich-Alexander-Universität Erlangen-Nürnberg (FAU), Erlangen, Germany shoulder angle [4] and adjustments of the infeed area [5] have already been identified. However, the influence of the material flow on the application relevant component properties was not investigated.

The field of operation ranges from high strength gears in power transmissions (e.g., vehicle gearboxes) to medium and low strength gears in low power transmissions (e.g., medical technology, food industry) [6]. For low loads, the metalplastic material pairing is suitable for use in dry-running gears, where the strength and accuracy requirements are significantly reduced [7]. The wide range of applications and performance of gears results in a broad demand for different gear materials. Besides, steel, non-ferrous metals, and light metals are applied [2]. The ready-to-use production of gears from these materials offers potential for low- and medium-loaded gears. However, existing investigations mainly address the cold forging of gears from steel materials [3]. Though, the mechanical properties of the gear material have a significant influence on the forming process [8]. Against this background, challenges arise when forming materials with varying flow strengths. The effect of the yield stress on the achievable application-relevant component properties has to be considered. In order to 
positively affect the process result, a basic understanding of the underlying mechanisms of action is required.

\section{Objective and methodology}

The aim of this investigation is to generate transferable process knowledge for the extrusion of gears from light and nonferrous metals in order to improve achievable component properties. For this purpose, the influence of the material on the process result and the underlying mechanisms of action are determined. Subsequently, tool-sided measures are developed and their effects on the component properties as well as relevant cause-effects are investigated. The gained knowledge is used to derive recommendations. Figure 1 shows the methodology within this investigation.

The three commonly applied gear materials $16 \mathrm{MnCr} 5$, $\mathrm{CuZn37}$, and AlMgSil were analyzed. The flow behavior of the materials and the tribological conditions during cold extrusion were determined in laboratory tests. Based on the obtained data, a numerical process model was built. The model was validated by conducting forming tests in order to ensure sufficient prediction quality. Numerical methods have already been applied by Jeong [4] and Kiener [9] for process analysis of gear cold-forging. Yet, these models were not used for a detailed investigation of the influence of the material yield stress and the elastic die deflection was not taken into account. With the help of the presented model, material-specific challenges regarding the achievable geometrical and mechanical component properties were identified. Subsequently, the underlying mechanisms of action were determined and suitable tool-sided measures were derived. These measures were numerically investigated and their cause-effects and influence on the relevant component and process properties were evaluated. Finally, transferable process knowledge was derived from the findings. When the application of a new gear material is requested, the findings regarding the yield stress influence can be transferred to identify material-specific challenges and derive appropriate tool-sided measures.

\section{Process modelling}

In order to achieve realistic modelling, the properties of the gear materials are characterized in upsetting tests and the lubrication systems are evaluated in double-cup-extrusion tests. The finite element (FE) models are built in the software simufact.forming 14.0.1. The gear manufacturing is carried out on a universal testing machine Schenck Trebel 400.
Fig. 1 Methodology within this investigation

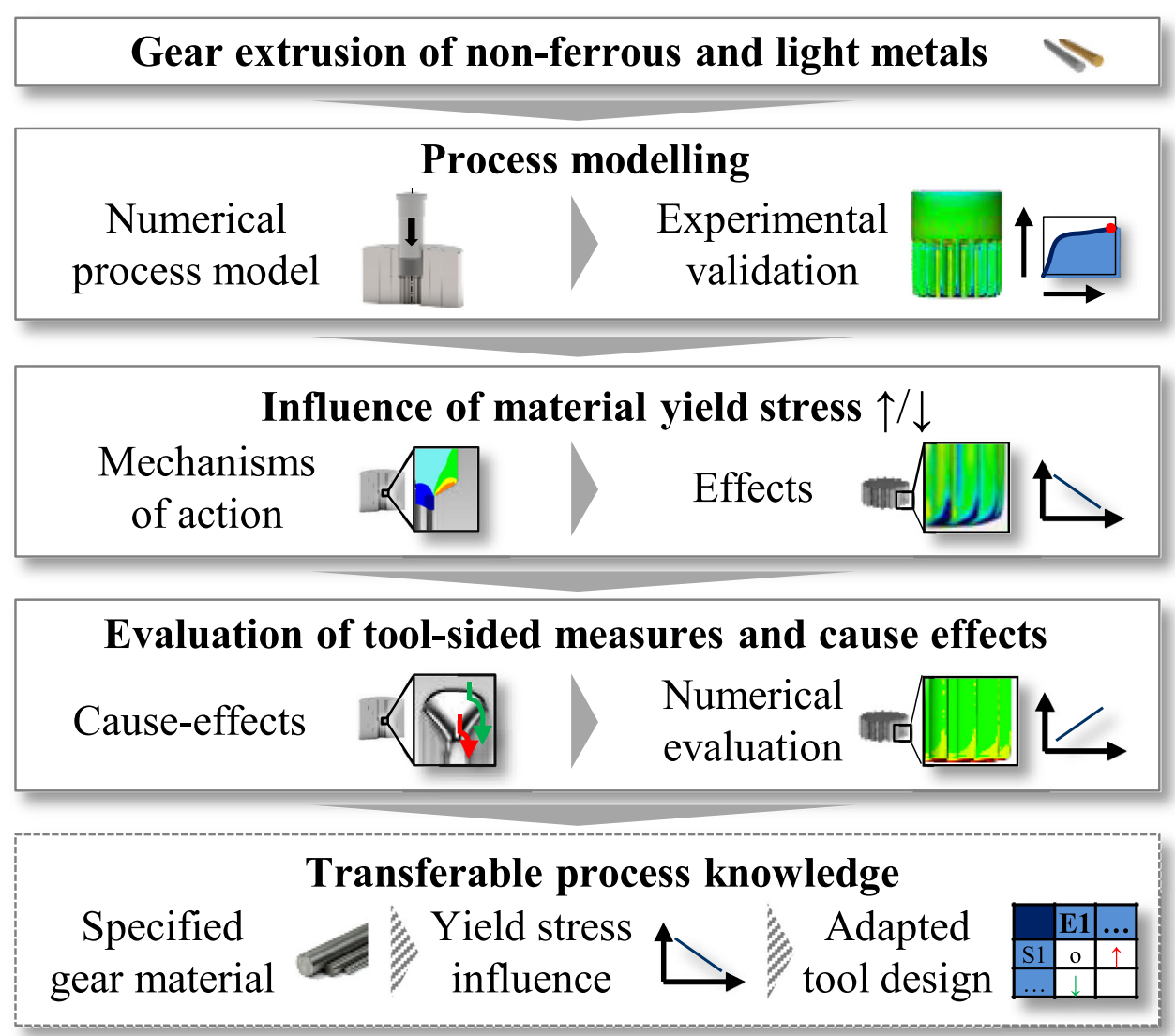




\subsection{Gear materials and lubrication systems}

Three different metallic materials are investigated, which cover a wide range of industrially relevant gear materials. The low-alloy case hardening steel $16 \mathrm{MnCr} 5$ (1.7131) is an frequently used gear material, usually applied for the production of gears with medium strength requirements [10] and can be case-hardened for higher demands. The brass alloy CuZn37 (2.0321) with a zinc content of about $37 \%$ has a homogeneous, face-centered cubic crystal structure, which is why it has very good cold formability [11]. Brass alloys are the most frequently non-ferrous metals used for gear manufacturing [2]. The heat treatable aluminum alloy AlMgSil (3.2315) is investigated as the third gear material. High-strength aluminum alloys are used for gears due to their good strength-toweight ratio [2].

\subsubsection{Flow behavior}

The flow properties were recorded in upsetting tests up to a true strain $(\varphi)$ of 0.8 at a deformation speed of $0.0067 \mathrm{~s}-1$ according to DIN 50106 (Fig. 2a). The experimentally determined flow curve was extrapolated to map higher strains as they occur during gear extrusion with the Hockett-Sherby approach [12]. This approach provides the best fit with the experimentally evaluated flow curves. In the range of the true strain from 1 to 3 , which occurs during gear manufacturing, the extrapolated flow curves show similar hardening behavior at significantly different stress levels. At a true strain of $\varphi=1$, the yield stress of $16 \mathrm{MnCr} 5$ is $915 \mathrm{MPa}$, whereas it is only $770 \mathrm{MPa}$ for CuZn37 and $371 \mathrm{MPa}$ for AlMgSi1.

\subsubsection{Tribological conditions}

In order to represent the tribological conditions realistically, the friction factors are determined in the double-cup-extrusion test (DCE test) [13]. For this purpose, a numerical process model of the DCE test was built in the simulation software simufact.forming 14.0.1 and experimental tests were carried out with the examined materials and lubricants. The occurring friction factors were evaluated by numerical identification from the cup height ratios determined in the experiment (Fig. 2b). All materials were formed with polymer-based lubrication systems. Due to the high contact pressures during the forming of $16 \mathrm{MnCr} 5$ caused by the high forming force required, a pretreatment was carried out by applying a phosphate layer [14]. To avoid direct tool contact and adhesive wear [15] during the forming of AlMgSi1, an aluminate layer was applied to the workpiece surface as a separating layer. The evaluated friction factors are $m=0.075$ for the steel material, $m=0.062$ for the brass alloy, and $m=0.039$ for the aluminum alloy. The highest friction occurs during forming of
Fig. 2 Evaluated flow curves and friction factors
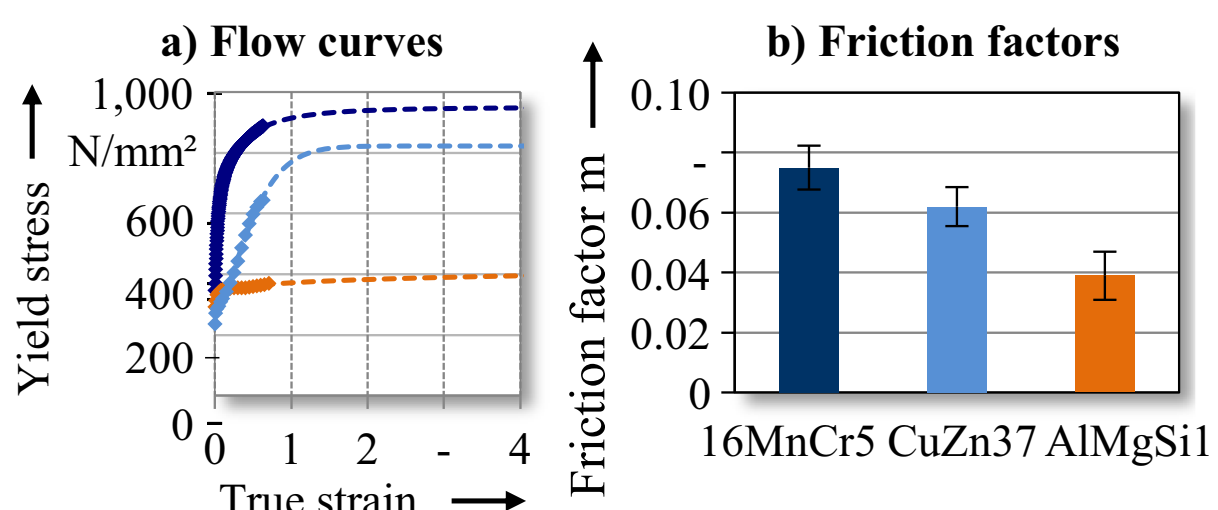

Compression test (DIN 50106) $\begin{aligned} \mathrm{n}_{\text {Samples }} & =10 \\ \mathrm{v}_{\text {Traverse }} & =5 \mathrm{~mm} / \mathrm{min}\end{aligned}$

\section{Data}

Compression test Extrapolation (Hockett Sherby)

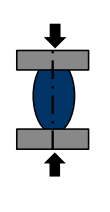

Double-cup-extrusion test (Geiger et. al)

$\mathrm{n}_{\text {Sample }}=10$

$\mathrm{v}_{\text {Punch }}=5 \mathrm{~mm} / \mathrm{min}$

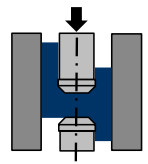

Material Pretreatment

$16 \mathrm{MnCr} 5 \square$ phosphate coating

CuZn37 none

AlMgSi1 aluminate coating 
the steel material, whereas the friction factor decreases for the brass and aluminum materials.

\subsection{Gear manufacturing}

In the following, the full forward extrusion of a spur gear is examined. The gearing has an involute profile with a normal module of 1 and a root radius factor according to DIN 780 of 0.3 [16]. In order to enable the manufacturing by full-forward extrusion, a tip radius of $0.3 \mathrm{~mm}$ is applied. Fig. 3 shows the tool system and the tooth geometry to be produced.

The geared area of the extrusion die is designed as a negative of the tooth geometry. The layout of the die and the design of the shoulder area are based on numerical preliminary investigations [17] and on literature recommendations [8]. Due to an expected internal pressure of more than 2000 $\mathrm{N} / \mathrm{mm}^{2}$, the die is reinforced twice [18]. In order to avoid stress peaks in the inlet area, the die is transversely split directly above the extrusion shoulder.

\subsection{Numerical process model}

The cold forging process is numerically investigated using the finite-element forming simulation software simufact.forming 14.0.1. The FE-model is built from the active tool components including the die, prestress rings, punch, ejector, and the workpiece. A coupled analysis of the material flow with elastic tool behavior is carried out, taking into account the radial prestressing applied by the reinforcement system. In the analysis, the elastic deflection of the die is calculated at any point in time within the process and its effect on the geometric shape of the formed gear is evaluated. The mechanical behavior of the workpiece material is assumed to be isotropic and strain rate-independent. In order to reduce the number of elements in the model and shorten calculation time, a segmented model was used (Fig. 4). A minimum segment angle of $62.5^{\circ}$ (3 teeth) was required in order to prevent meshing problems due to a too steep angle in the center area of the workpiece.

The workpiece is meshed with hexahedrons to accurately map the three-dimensional deformation [19]. The tool components are discretized with tetrahedrons, which reduces the computing time in comparison with hexahedrons and provide sufficient results considering the low deformation of the tools [19]. The required resolution of the FE mesh for a sufficiently accurate reproduction of the gearing accuracy according to the ISO tolerance classes [20] was determined in preliminary investigations. The edge area of the workpiece in which the tooth profile is formed is meshed with a minimum edge length of $0.10 \mathrm{~mm}$. In the inner area of the workpiece, the mesh is coarsened in order to reduce the calculation effort. Accordingly, the geared area of the die is finely meshed $(0.10 \mathrm{~mm})$ and the edge area and the reinforcement rings are coarsely meshed $(1.50 \mathrm{~mm})$. In total, the die is meshed with 211,686 elements and the workpiece with 191,021 elements. The workpiece is remeshed if the elongation exceeds $40 \%$. The simulation is divided into 1000 incremental steps.

\subsection{Experimental validation}

The validation of the numerical process model was carried out by comparing the numerically determined process and component properties with those of experimentally produced components. The force-displacement curve is measured by an incremental encoder (resolution $3.3 \mu \mathrm{m}$ ) and a force sensor (accuracy $0.5 \%$ ) directly on the traverse of the forming press. Clearance and rigidity of the tool and press are compensated by a recorded correction curve. A comparison of the process force progression is shown in Fig. 5.

With regard to the force-displacement curve, there is a good agreement with deviations of less than $3 \%$ for all three materials. A more in-depth analysis of the prognosis
Fig. 3 Gear manufacturing by full forward extrusion
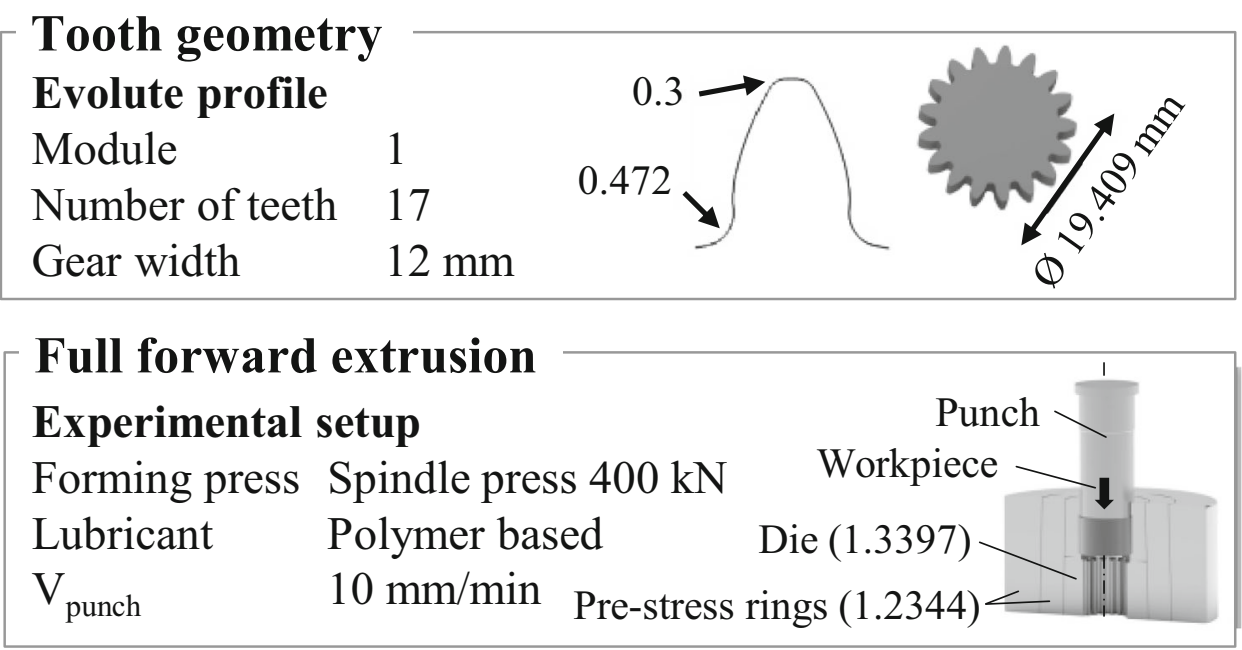
Fig. 4 Numerical modelling of cold forging process
Segmentation

$63.5^{\circ}$

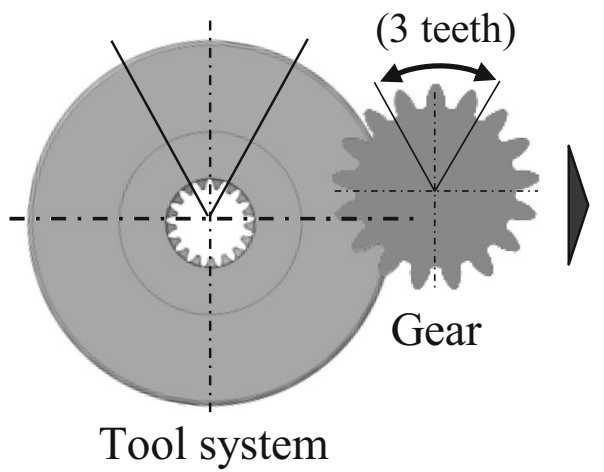

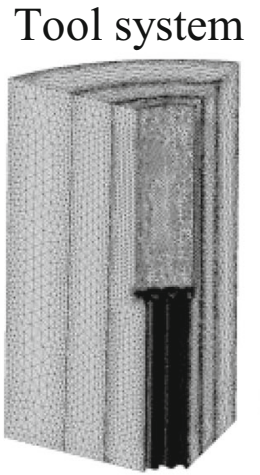

Element size
Meshing

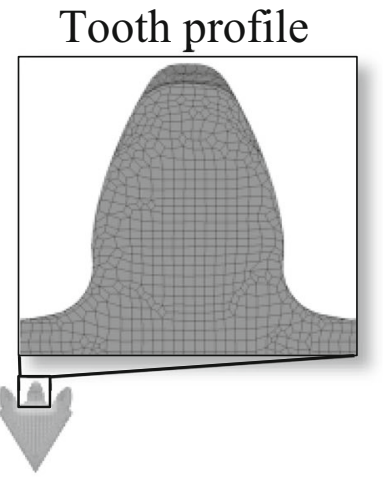

Element size
$0.1-1.5 \mathrm{~mm}$
$0.1 \mathrm{~mm}$

capability is carried out by evaluating the geometrical (Fig. 6) and mechanical (Fig. 7) component properties.

The geometry of the experimentally produced components was recorded with a stripe light scanner $(G O M G m b H)$ in order to evaluate the overall die filling. Fig. 6a shows a comparison of the experimental and numerically determined geometry. The evaluation shows good agreement over the entire component height for all three materials.

The classification of the achieved tolerance class is based on the determination of the total profile and total flank line deviations according to ISO 1328 [20]. The profile accuracy is measured in the transverse section at medium height of the gearing and provides information on the range of the minimal and maximal deviation of the involute tooth profile from the nominal geometry. The flank line accuracy is determined over the gearing width accordingly. In Fig. 6b, the numerically determined profile and flank line accuracies are compared with the measurement of manufactured components by a coordinate measuring device. Regarding the profile accuracy, a very good agreement is achieved and the simulation correctly determines the resulting tolerance classes of $16 \mathrm{MnCr} 5$ (IT 7), CuZn37 (IT 6) and AlMgSil (IT 5). Furthermore, the simulation very well predicts the flank deviation of the material $16 \mathrm{MnCr} 5$. For the materials $\mathrm{CuZn} 37$ and AlMgSi1, high deviations are determined equally in experiment and simulation. Overall, however, there is less good agreement for these materials. This is attributed to the increased insufficient die filling in the lower area of the gear, which has a negative effect on the flank deviation determined over the tooth width.

In addition to the geometric component properties, the mechanical properties are evaluated in order to determine the prediction quality of the simulation model. In Fig. 7, the
Fig. 5 Comparison of process forces from experiment and simulation

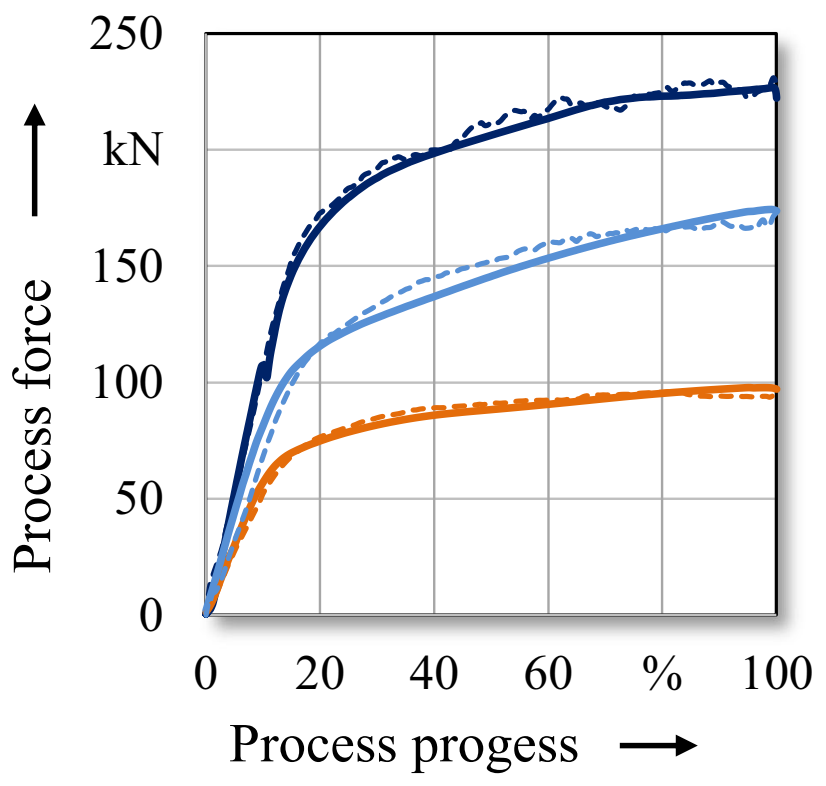

\section{Gear extrusion}

$\mathrm{v}_{\text {Punch }}=10 \mathrm{~mm} / \mathrm{min}$

$\mathrm{n}_{\text {Gears }}=10$
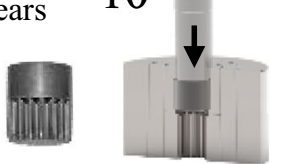

Experiment

Simulation

Gear material

$16 \mathrm{MnCr} 5-$

CuZn37

AlMgSi1 
Fig. 6 Comparison of geometrical component properties from experiment and simulation a) Geometry

Experimental workpiece
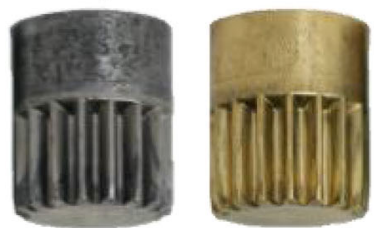

$-5 \mathrm{~mm}$

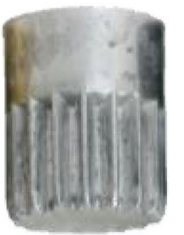

Comparison of experiment and simulation

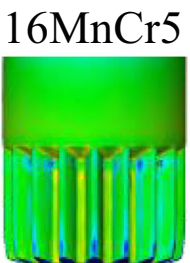

CuZn37

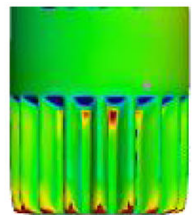

AlMgSi1

$-5 \mathrm{~mm}$ b) Gearing accuracy Profile $\triangle$
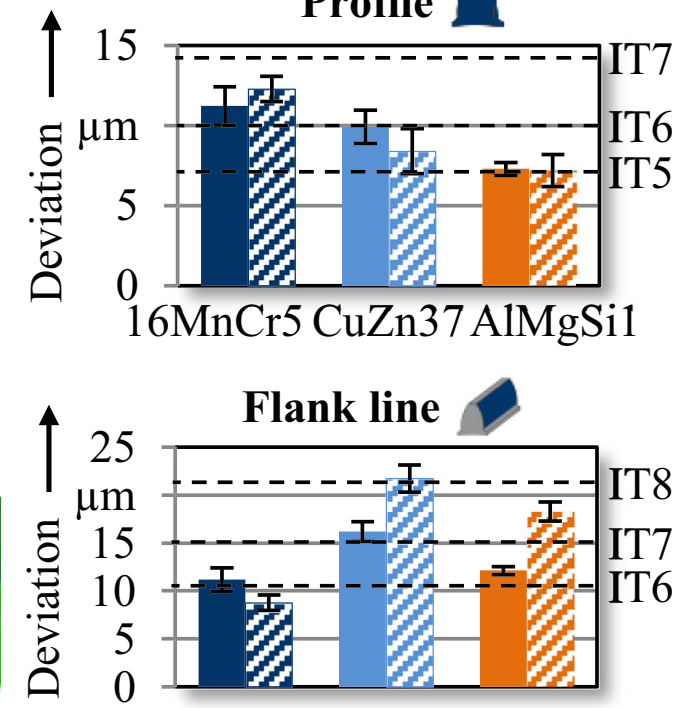

16MnCr5 CuZn37 AlMgSi1

\begin{tabular}{|c|c|c|}
\hline $\begin{array}{l}\text { Surface comparison } \\
\text { Deviation } \\
-0.1 \quad 0.0+0.1 \mathrm{~mm}\end{array}$ & $\mathrm{n}_{\text {Gears }}=3$ & $\begin{array}{l}\text { Gearing accuracy } \\
\text { (ISO 1328) } \\
\text { Experiment } \\
\text { L Simulation }\end{array}$ \\
\hline
\end{tabular}

microhardness of the experimental formed gears is compared with the true strain in the simulation.

The resulting strain hardening of the formed tooth profile was measured by means of the microhardness (HV 0.02, test force $200 \mathrm{mN}$ ) at the medium gear width. The measurement reveals a strong hardening of the surface of the tooth profile, especially in the area of the tooth flank and root. This hardening is caused by plastic deformation during forming [21]. In the simulation, the plastic strain can be evaluated in order to estimate the resulting strain hardening. For the investigated materials, the comparison of the microhardness from the experiment and the plastic strain from the simulation provides a good qualitative prediction of the hardness distribution.

Fig. 7 Comparison of mechanical component properties from experiment and simulation
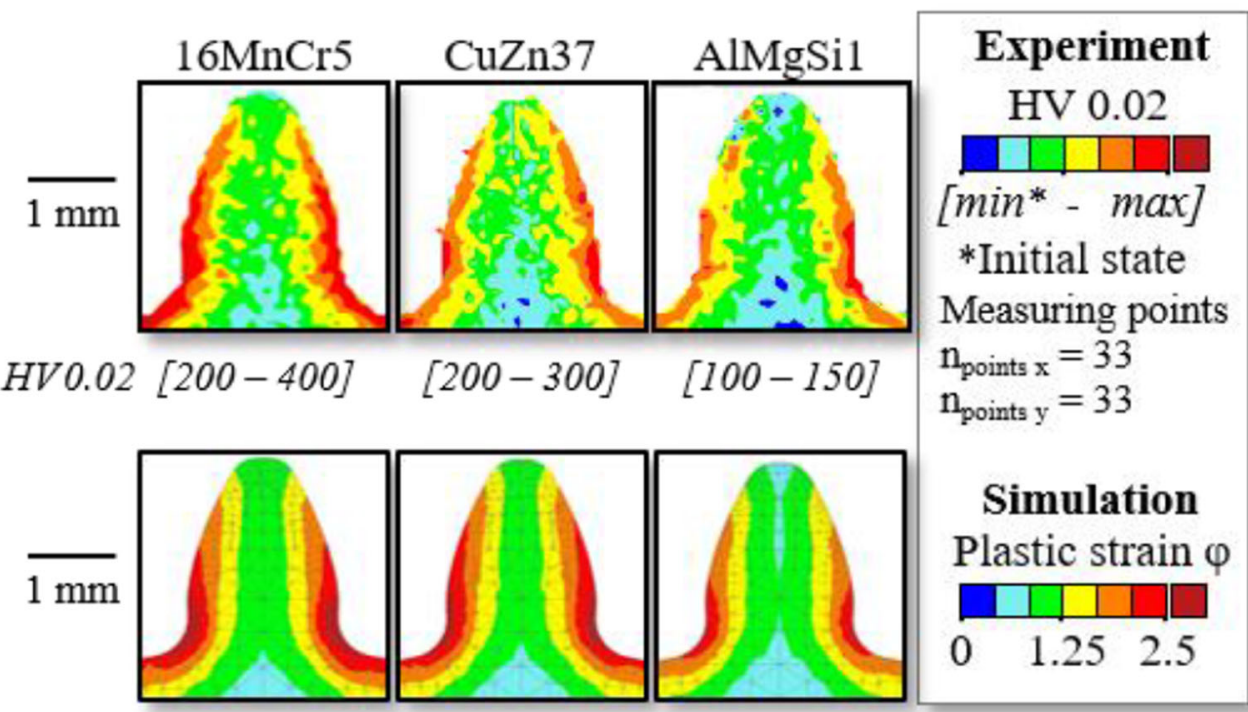
The comparison of the process and component properties between simulation and experiment shows a very good overall prediction quality for the gear extrusion of all three materials. Against this background, the numerical process model is used for the investigation in the further course of this research.

\section{Identification of material-specific challenges}

The material represents one of the most important parameters in gear drive design. At the same time, when applying forming processes, the material has a fundamental influence on the material flow and thus on the achievable component and process properties.

\subsection{Relevant component and process properties}

As relevant component properties, the achievable die filling, gearing accuracy, and strain hardening of the tooth profile are evaluated. Regarding the process properties, the maximum process force is investigated. In the following, the evaluated component and process properties are described (Fig. 8).

\subsubsection{Die filling}

The high material utilization represents a major advantage for the application of cold forming processes. However, during cold extrusion of gears, insufficient die filling poses a key challenge. The usable height and resulting material efficiency are limited due to two insufficiently filled areas in the lower and upper ends of the workpiece [4]. At the beginning of the forming process, due to low resistance when entering the unfilled die, the material flows axially ahead. This leads to a decreased tool contact of the material in the feed area and insufficient die filling (inflow area) in the lower part of the gear. Furthermore, an impression of the extrusion shoulder of the die remains in the upper part of the workpiece. The height of this shoulder imprint depends on the geometry of the shoulder area of the extrusion die. In the context of this investigation, a deviation of more than $0.1 \mathrm{~mm}$ between the formed tooth profile and the nominal profile is evaluated as insufficient die filling. With this deviation, a maximum gearing accuracy of IT Class 12 according to ISO 1328 can be achieved at most [20]. In the case of larger deviations, it is assumed that the gearing is no longer functional.

\subsubsection{Gearing accuracy}

With regard to compliance with installation and connection dimensions as well as a smooth running behavior and load capability, a sufficient gearing accuracy according to the tolerance specification is required [22]. As essential parameters for the gearing accuracy, the total profile and flank line deviations are evaluated according to ISO 1328 [20].

\subsubsection{Strain hardening}

The plastic deformation in the forming process causes increased hardness of the tooth profile, which has a positive effect on the mechanical load capacity of the tooth root and the wear resistance of the tooth flank [23]. Against this background, the strain hardening of the tooth head, tooth flank, and tooth root is evaluated.

\subsubsection{Process force}

The maximum process force level is evaluated as relevant process characteristics. In particular, changes of the maximum force requirement provide information about changes in the material flow during the forming process. The maximum process force has an effect on the occurring tool load and resulting tool deflection. It also provides information on the energy requirement and energy efficiency of the forming process.

\subsection{Influence of workpiece material and underlying mechanisms of action}

During extrusion of gears from materials with varying yield stress, material-specific challenges arise. The underlying mechanisms of action are determined with help of the validated numerical process model. Subsequently, they are confirmed by experimental results. In the following, the influence of the gear material on the achievable die filling is investigated (Fig. 9).

Fig. 9a shows the material flow velocity at the beginning of the process (20\% process progress) during forming of $16 \mathrm{MnCr} 5$ (yield stress of $915 \mathrm{MPa}$ at $\varphi=1$ ) as well as
Fig. 8 Evaluated component and process properties

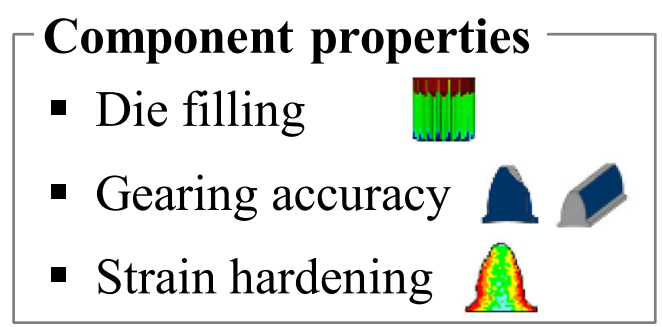


Fig. 9 Influence of the gear material on the achievable die filling

\section{a) Mechanism of action}
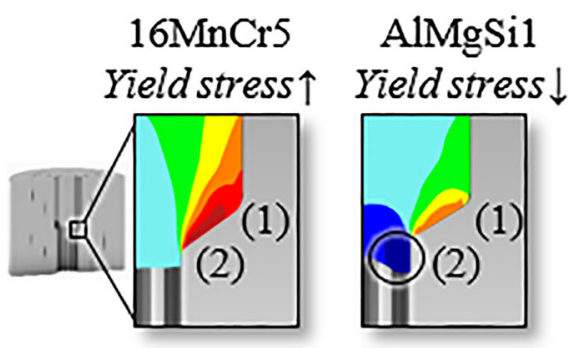

(1) Forming zone

(2) Inflow area

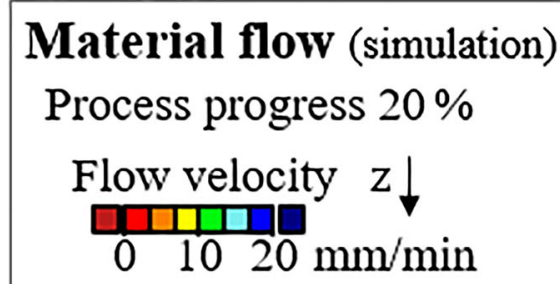

b) Experimental verification
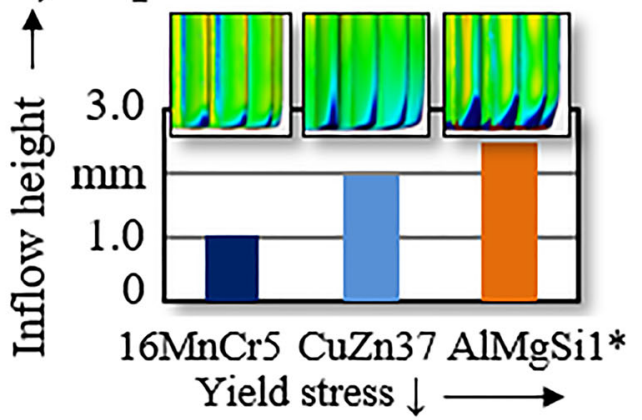

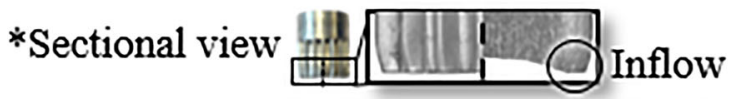

Die filling (experiment, $\mathrm{n}=5$ ) Inflow height $h_{I}$

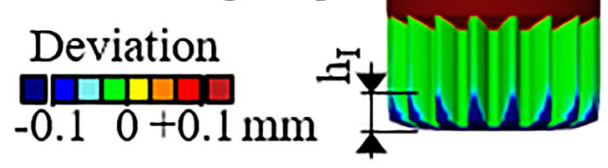

AlMgSil (371 MPa at $\varphi=1$ ). When the steel material with a comparatively high yield stress is formed, the material flow in the shoulder area (1) of the die is decelerated due to the high flow resistance, which results in a locally reduced material flow velocity compared with the inflow area (2). In contrast to that, the lower yield stress of the aluminum material leads to a lower flow resistance in the forming zone and a significantly increased local material flow velocity. This reduces tool contact and causes the material to flow axially ahead into the unfilled geared area of the die (2), leading to insufficient die filling.

The resulting material inflow in the middle of the component is visible in the sectional view of the formed aluminum gear (Fig. 9b). From the experimental data, it can be seen that with lower yield stresses, due to the reduced tool contact at the beginning of the process, the height of the inflow area is increased and the die filling is decreased. For $16 \mathrm{MnCr} 5$, the inflow height is $1.05 \mathrm{~mm}$ and the material utilization is $82.1 \%$. The inflow height increases to $1.95 \mathrm{~mm}$ for CuZn37 and further to $2.47 \mathrm{~mm}$ for AlMgMn1. The material utilization is decreased to $75.2 \%$ and $71.5 \%$.

In addition to the die filling, the accuracy of the tooth profile is a decisive factor for the evaluation of the geometry [20]. Fig. 10 shows the effect of the material yield stress on the achievable gearing accuracies based on the profile deviation.

The deviation of the extruded tooth profile from the nominal geometry can mainly be attributed to the elastic deformation of the extrusion die during forming. The deflection leads to a radial displacement of the die tooth profile and an excess of the extruded tooth geometry. The radial displacement that occurs during forming of the different materials is shown in Fig. 10a. An elevated force requirement due to a higher yield stress leads to an increased radial elastic deflection of the extrusion die, which has a negative impact on the achievable accuracy of the tooth profile. The largest radial deflection of $15.70 \mu \mathrm{m}$ occurs during forming of $16 \mathrm{MnCr} 5$, which also requires the highest forming force $(228 \mathrm{kN})$. In contrast to that, the radial displacements during forming of $\mathrm{CuZn} 37$ $(168 \mathrm{kN})$ and AlMgSil $(88 \mathrm{kN})$ are significantly lower at $12.30 \mu \mathrm{m}$ and $6.10 \mu \mathrm{m}$.

Due to the reduced tool deflection, smaller profile deviations are achieved when forming materials with lower yield stress (Fig. 10b). For gears extruded from $16 \mathrm{MnCr} 5$, the total profile deviation is $12.30 \mu \mathrm{m}$ (IT class 7). When forming $\mathrm{CuZn37}$, this is reduced to $9.90 \mu \mathrm{m}$ (IT class 6) and for further to AlSi1MgMn to $7.20 \mu \mathrm{m}$ (IT class 5).

Besides the geometry, the mechanical properties are of importance regarding the operational behavior of the gear. The forming induced hardening influences the mechanical load capacity of the tooth root and the wear resistance of the tooth flank. Fig. 11a shows the influence of the yield stress on the resulting work hardening when forming different materials.

The characterization of the tribological conditions in Fig. 2 indicates decreasing friction factors for materials with lower yield stress. These are attributed to the reduced contact pressure during forming of softer materials (Fig. 11a). High contact pressures influence the performance of the lubricant layer and lead to increased friction factors 
Fig. 10 Influence of the gear material on the achievable profile accuracy a) Mechanism of action $16 \mathrm{MnCr} 5$ AlMgSi1 Yield stress $\uparrow \quad$ Yieldstress $\downarrow$ Process force $\uparrow$ Process force $\downarrow$

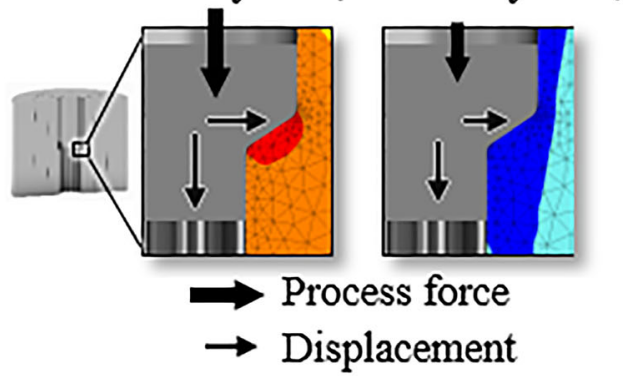

Die deformation (simulation) Radial displacement पाIII)

$0 \mu \mathrm{m} 20$

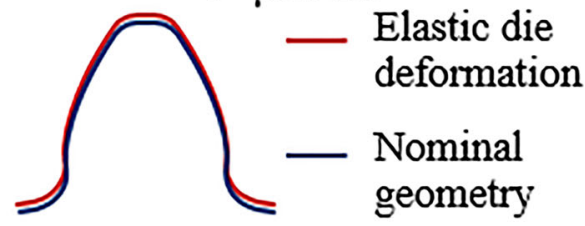

b) Experimental verification

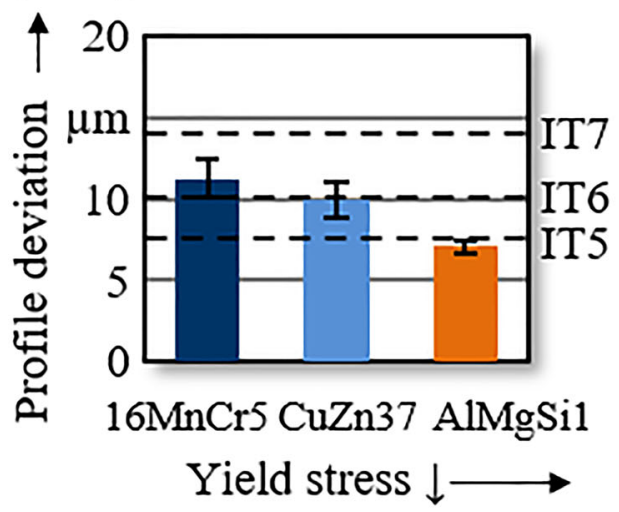

Accuracy (experiment, $n=3$ )

Profile deviation $\_$(ISO1328)

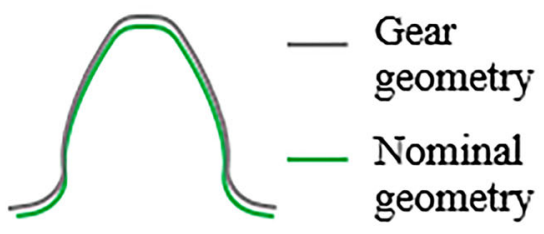

[14]. A friction factor of $m=0.075$ occurs during forming of $16 \mathrm{MnCr} 5$. In contrast to that, the friction factor during forming of AlMgSil with a significantly lower yield stress is only $m=0.039$. The reduced friction decreases the degree of deformation and hardening of the tooth surface
(Fig. 11b). When forming $16 \mathrm{MnCr} 5$, average true strains of 2.64 occur in the tooth root. Compared with that, the plastic strain when forming CuZn37 is only 2.45 and with AlMgSi1 2.21. For a reasonable comparison of the process-induced hardening of the experimental produced
Fig. 11 Influence of the gear material on the resulting work hardening

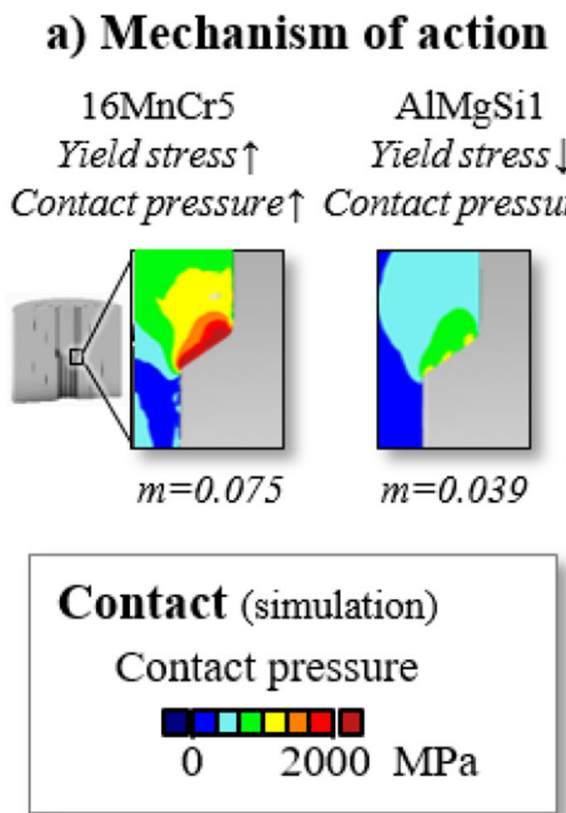

b) Experimental verification

Yield stress $\downarrow \longrightarrow$

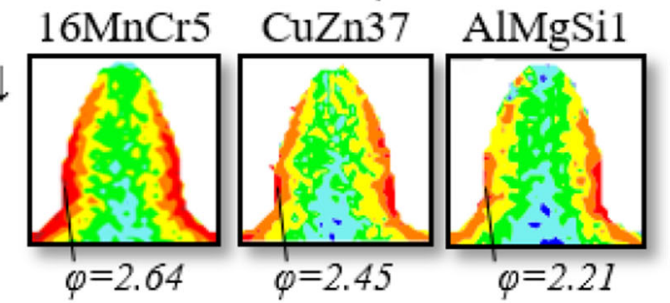

$H V[200 *-400][180 *-300][100 *-150]$

Hardening $\downarrow$

Hardening (experiment, $\mathrm{n}=1$ ) HV 0.02 Measuring points $\mathrm{n}_{\text {points } \mathrm{x}}=33$ [initial* $\left.{ }^{*} \max \right] \mathrm{n}_{\text {points }}=33$ 
components, the measured microhardness was put in relation to the corresponding initial hardness of the material. With respect to the initial and maximum hardness, the brass and aluminum material show a lower hardening compared with the steel material, which corresponds to the numerically determined degrees of deformation.

\section{Analysis of tool-side measures to influence the component properties}

In Section 4, the influence of the yield stress of the gear material on the extrusion process was determined and challenges were identified. In the following, tool-sided measures are derived in order to meet these challenges and to improve the application-relevant component properties of the gearing. With the help of the validated, numerical process model, the measures are investigated and the underlying cause-effects are evaluated.

\subsection{Derivation of tool-sided measures}

Essential factors influencing forming processes are the workpiece, the lubrication system, and the forming tool [8]. The influence of the workpiece is mainly affected by the yield behavior of the gear material, which is predetermined by the later application. At the same time, the use of different materials also requires material-specific lubrication systems with varying performance (Fig. 2). This is why the lubrication system does not offer a generally applicable method to influence the process result when forming different materials.

With a given material and lubrication system, the tool geometry can be used as a universal control lever to influence the material flow during forming and to improve the achievable component and process properties. The main objectives are listed below:

- Improved die filling

- Increased gearing accuracy

- Elevated strain hardening of the tooth root and flank

The design of the forming zone affects the material flow. Fig. 12 shows the developed tool-sided control levers and the underlying mechanisms.

By adjusting the shoulder angle, the deflection of the material flow in the shoulder area is strongly affected. The aim is to reduce the material flow speed globally in order to achieve increased die contact and improved die filling at the beginning of the process.

The application of a flank angle causes an additional deflection of the material flow, which is directed to the tooth root and flank area. This is intended to increase die filling and work hardening in these application relevant areas.

The objective of an increased infeed radius is to a locally improve material flow by increasing the radius in the die tooth root and flank area compared with the radius in the head area of the die tooth. This is supposed to change the local plastic strain and the resulting hardness distribution of the tooth profile.

\subsection{Numerical analysis and explanation of cause- effects}

As reference geometry for this analysis, a tool geometry with a shoulder angle of $120^{\circ}$, no flank angel $\left(0^{\circ}\right)$, and an infeed radius of $0.3 \mathrm{~mm}$ has been used. For each tool modification, three variations within the minimum and maximum possible range are examined (Fig. 13). These are limited by the occurring tool load as well as by constructive possibilities.
Fig. 12 Investigated tool-sided measures and their mechanisms of action

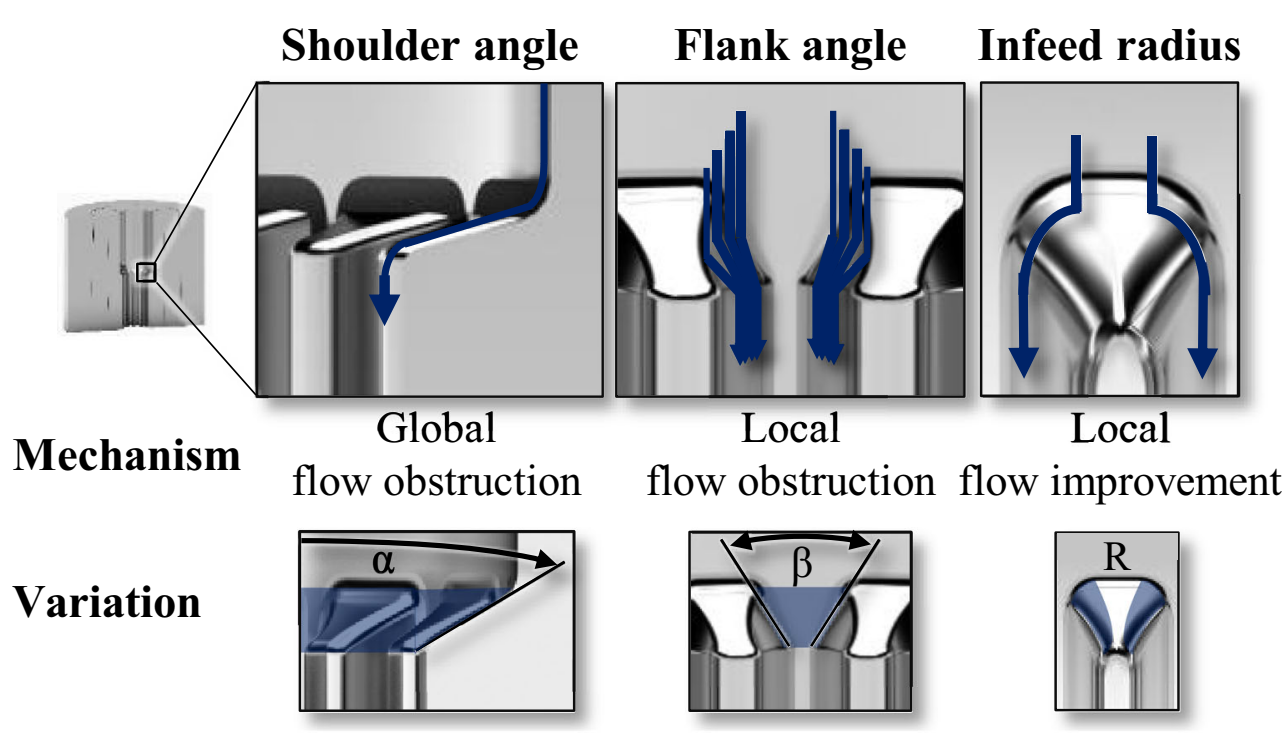


Fig. 13 Investigated variations of the tool geometry and their effect on the material flow a) Shoulder angle

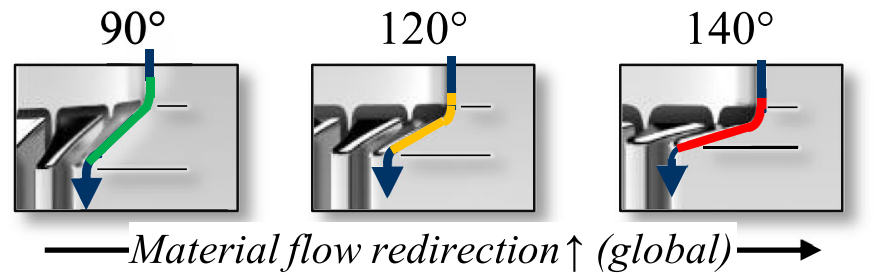

b) Flank angle
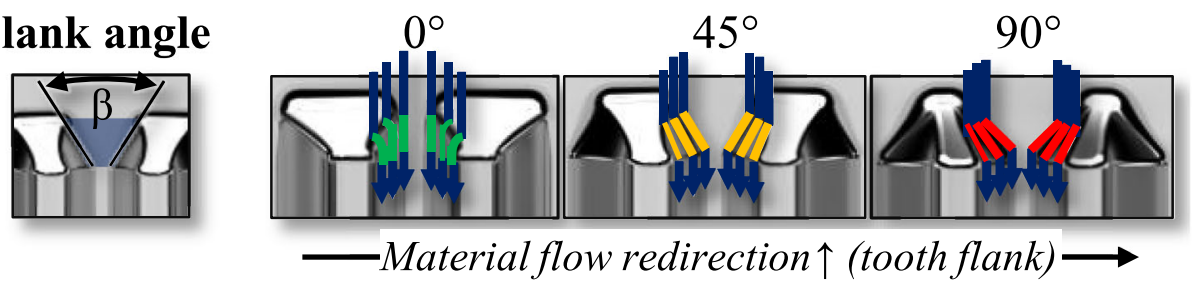

c) Infeed radius

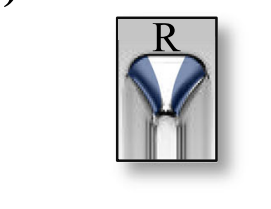

$0.3 \mathrm{~mm}$
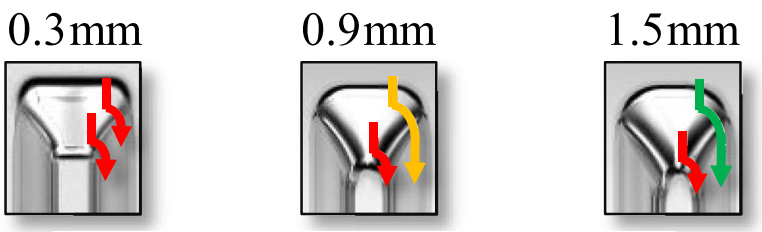

- Material flow improvement $\uparrow$ (local) $\rightarrow$

Materialflow - low - medium - high deflection

\subsubsection{Variation of shoulder angle (global flow obstruction)}

An increase of the shoulder angle leads to an extended redirection of the material flow and a smaller forming zone (Fig. 13a). Since the global forming speed is constant, the material flow velocity is reduced locally in the shortened forming zone. The reduced flow velocity leads to improved tool contact, which positively affects die filling. However, the required forming force is increased due to the elevated material flow deflection. Preliminary numerical investigation have shown insufficient die filling $(<70 \%)$ for angles smaller than $90^{\circ}$ as well as critical tool loads for angles above $140^{\circ}$. This is why shoulder angles of $90^{\circ}$ and $140^{\circ}$ were investigated in addition to the reference of $120^{\circ}$.

\subsubsection{Variation of flank angle (local flow obstruction)}

The integration of a flank angle causes an additional redirection of the material flow into the area of the tooth root and flank (Fig. 13b). This leads to increased deflection. Flank angles of $0^{\circ}$ (reference), $45^{\circ}$, as well as the maximum possible angle of $90^{\circ}$ - which is limited due to critical occurring tool loads - are examined.

\subsubsection{Variation of infeed radius (local flow improvement)}

An enlargement of the infeed radius results in a smoother material deflection in the root area of the die tooth (Fig. 13c). In contrast, the forming zone in the area of the die tooth head is limited due to the low tooth thickness in this area. This leads to varying levels of material deflection and flow speed in the head and root area of the tooth profile.

\subsubsection{Effects on component and process properties}

In the following, the effects of the adapted material flow on the process and component properties of gears extruded from AlMgSil (3.2315) are presented. For this purpose, the validated numerical process model is used. Changes in the maximum force requirement provide information about changes of the material flow during the forming process. The maximum process force is of importance regarding the occurring tensile stress and the resulting tool deflection. The required maximum process forces are shown in Fig. 14.

The deflection of the material flow, which increases with an enlargement of the shoulder angle (Fig. 13a), causes an elevated required forming force. Compared with a shoulder angle of $120^{\circ}$, where a maximum process force of $87.67 \mathrm{kN}$ is required, the force is reduced to $81.60 \mathrm{kN}(-7 \%)$ for an angle of $90^{\circ}$ and increased to $91.60 \mathrm{kN}(+12 \%)$ for a maximum shoulder angle of $140^{\circ}$.

An additional material deflection is also caused by application of the flank angle. It is directed to the tooth flank area (Fig. 13b) and can be recognized by slightly increased process forces. Against a process force of $87.67 \mathrm{kN}$ at a flank angle of $0^{\circ}$, the process forces rise to $88.32 \mathrm{kN}\left(45^{\circ}\right)$ and $89.40 \mathrm{kN}$ $\left(90^{\circ}\right)$. 
Fig. 14 Numerical investigated influence of tool-sided measures on the required process force

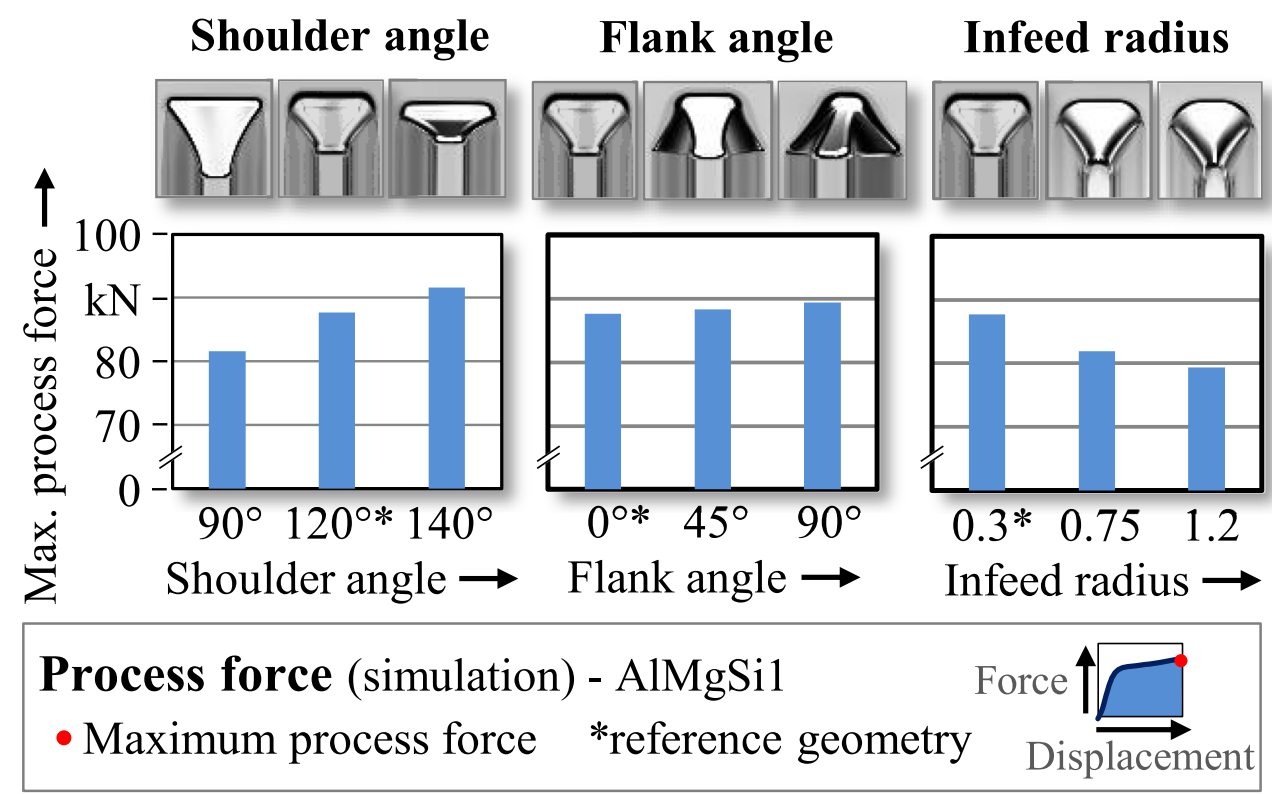

In contrast to that, a reduced material redirection is achieved by increasing the infeed radius. The material flow is less strongly deflected in the root area of the die tooth (Fig. 13c) leading to an overall reduced force requirement and a reduction in the maximum process force of $87.67 \mathrm{kN}$ in the reference process (infeed radius $0.3 \mathrm{~mm}$ ) by $10 \%$ to $79.40 \mathrm{kN}$ for an inlet radius of $1.2 \mathrm{~mm}$.

In the following, it is examined how the adapted material flow affects the die filling and thus the material utilization.
Fig. 15 shows the influence of the tool-sided measures on the achievable die filling.

An enlargement of the shoulder angle causes in an extended redirection of the material flow and a shortened forming zone (Fig. 13a). This leads to a locally reduced flow velocity and increasing tool contact in the forming zone, which reduces the material inflow height. Furthermore, a reduced height of the geometry-related shoulder imprint results from the shortened forming zone. This
Fig. 15 Numerical investigated influence of tool-sided measures on die filling

\section{Shoulder angle Flank angle}
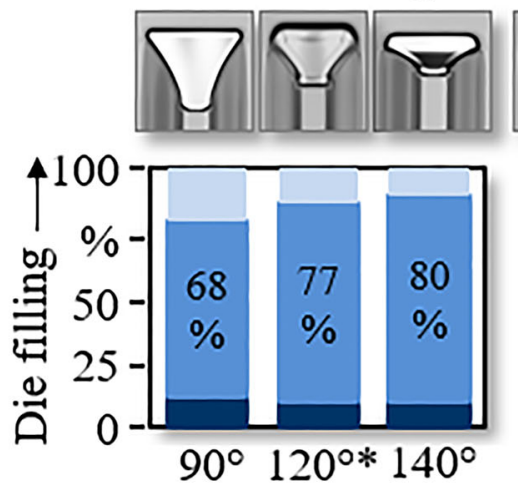

Shoulder angle $\rightarrow$
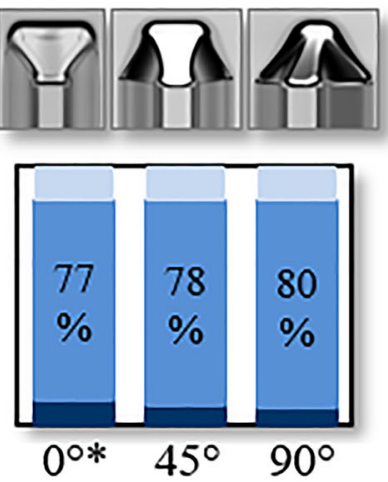

Flank angle $\rightarrow$
Infeed radius
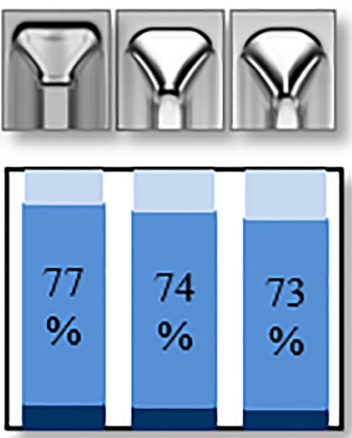

$0.3 * 0.75 \quad 1.2$
Die filling (simulation) - AlMgSi 1
Shoulder imprint
$\square$ Useable height
$\square$ Inflow height
*reference geometry
Deviation
$-0.10+0.1 \mathrm{~mm}$

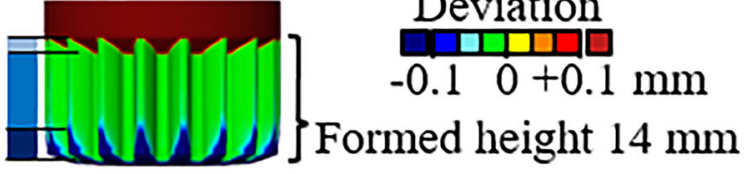


improves overall die filling by increasing the shoulder angle to $140^{\circ}$; the achievable die filling is improved to $80 \%$.

The additional deflection of the material flow at the flank angle ( Fig. 13b) enhances tool contact and improves the die filling. The maximum possible flank angle of $90^{\circ}$ increases the die filling to $80 \%$.

An increase of the infeed radius results in a lower material flow deflection in the root area of the die tooth and thus an enlarged material flow velocity in this area (Fig. 13c). This has a negative effect on tool contact at the beginning of the process, reducing the achievable die filling in the inflow area and the usable height of the gearing.

In addition to the die filling, the achievable accuracy is important for the production of ready-to-use gears. Fig. 16 shows the influence of the tool-sided measures on gearing accuracy.

The stronger deflection of the material flow with increasing shoulder angle (Fig. 13a) leads to a higher forming force and elevated elastic expansion of the extrusion die. Due to the higher deflection of the tooth profile, the forming accuracy is reduced and the profile deviations are increased. In comparison with a shoulder angle of $120^{\circ}$, where profile deviations of $7.20 \mu \mathrm{m}$ (IT 6) are achieved, they are increased to $8.40 \mu \mathrm{m}$ at a shoulder angle of $140^{\circ}$, whereas they are reduced to $6.85 \mu \mathrm{m}$ (IT 5) at an angle of $90^{\circ}$.

However, significantly higher deviations and worse tolerance classes are achieved regarding the flank line accuracy. The increased redirection of the material flow with steeper shoulder angle improves the die filling over the gear width, as already shown in Fig. 15. This results in a significant improvement of the flank line accuracy. At a shoulder angle of $120^{\circ}$, the total flank line deviation is $18.32 \mu \mathrm{m}$ (IT 8). It is reduced to $12.50 \mu \mathrm{m}$ (IT 7) at an angle of $140^{\circ}$. In contrast to that, an angle of $90^{\circ}$ leads to a total flank line deviation of $23.00 \mu \mathrm{m}$ (IT 9). By adjusting the material flow in the shoulder area, the achievable gearing accuracy is improved significantly.

Since the process forces are changed only marginally by changes of the flank angle and the infeed radius, the tool deflection and achievable profile deviation are not affected. As furthermore the die filling is only slightly influenced, there is also no significant effect on the accuracy of the flank line. With regard to the operational behavior of the gearing, the mechanical properties are important as well. Fig. 17 shows the influence of the measures on the hardening of the tooth profile.

With a steeper shoulder angle, the increased material flow deflection (Fig. 13a) leads to elevated plastic strain and resulting work hardening in all areas of the tooth profile. In the areas of tooth flank and root relevant to the application, true strains of $2.78(+38 \%)$ and $3.76(+32 \%)$ are achieved at a shoulder angle of $140^{\circ}$.

The application of a flank angle leads to an additional deflection of the material flow into the area of the tooth flank (Fig. 13b). Thus, the plastic strain in these areas is increased. Compared with the reference (flank angle $0^{\circ}$ ), a flank angle of $90^{\circ}$ leads to an increase in the true strain in the tooth flank to $2.15(+5 \%)$ and to $3.95(+40 \%)$ in the tooth root. This improves the mechanical load capacity of the gearing.

Due to an increase of the infeed radius, a smoother deflection of the material flow results in the root area of the die teeth, where the head and flank of the toothing are formed. In contrast to this, the comparatively small radius at the tooth head of the die teeth, due to the small remaining tooth thickness, leads to a stronger material deflection in this area (Fig. 13c). The
Fig. 16 Numerical investigated influence of tool-sided measures on profile and flank line accuracy
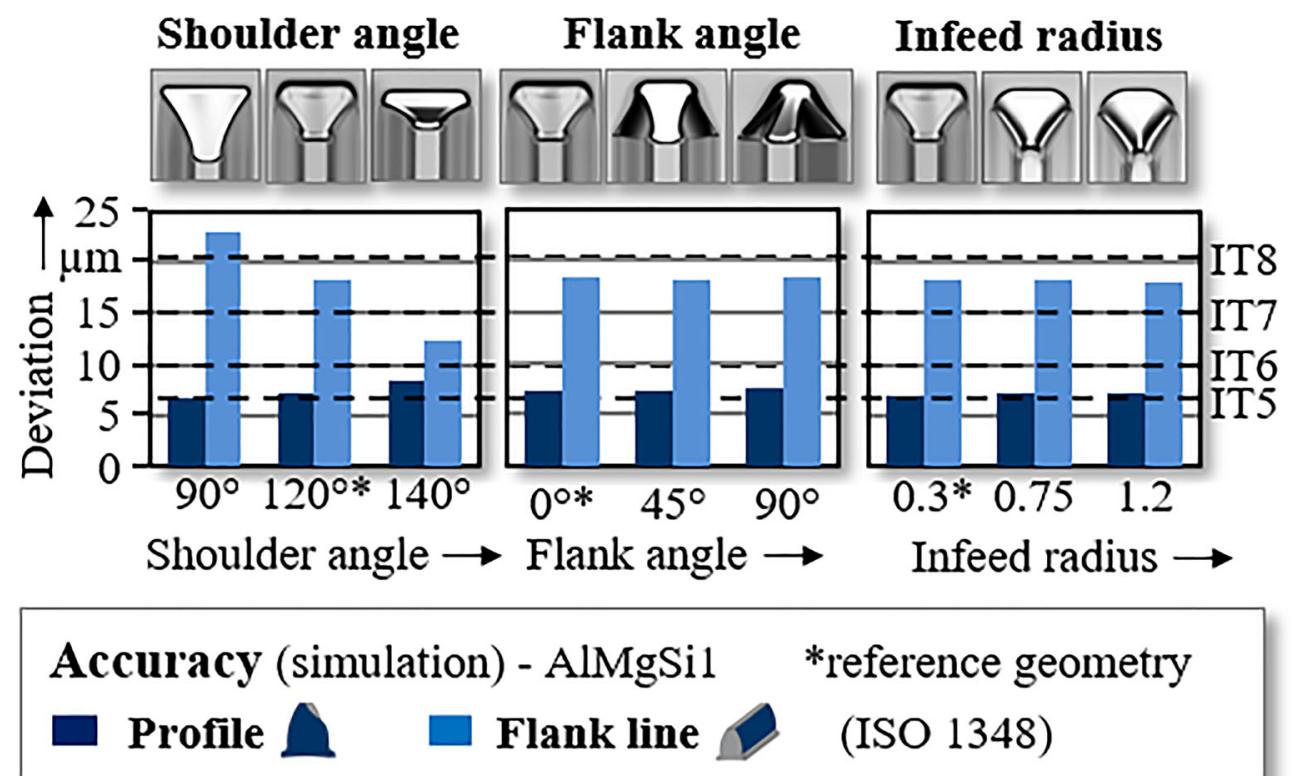
Fig. 17 Numerical investigated influence of tool-sided measures on strain hardening

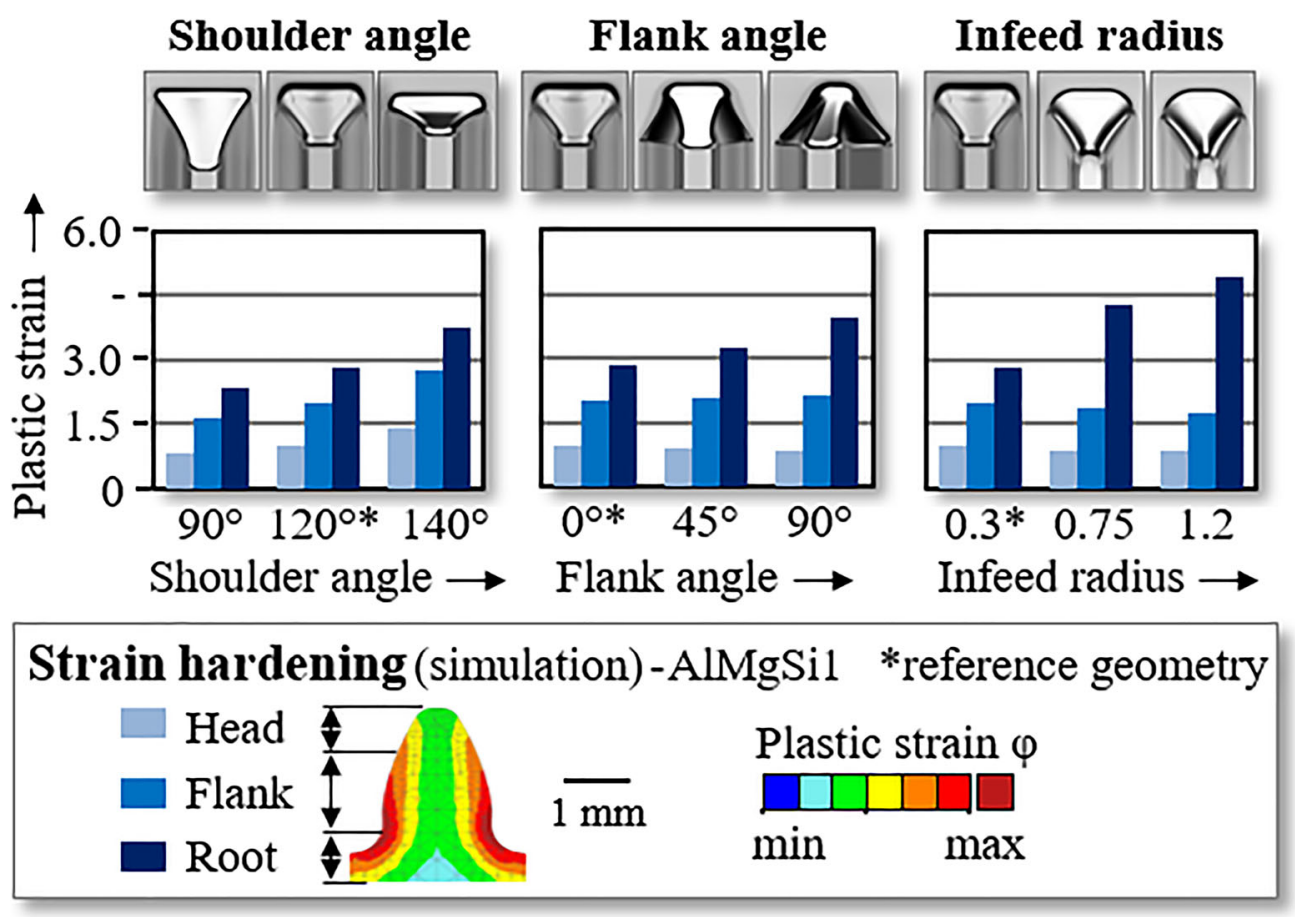

true strain in the tooth root of the extruded toothing, which is formed in the head area of the die toothing, is increased by $74 \%$ from 2.84 at an infeed radius of $0.3 \mathrm{~mm}$ (reference) to 4.95 at an infeed radius of $1.2 \mathrm{~mm}$. The true strain of the tooth tip and the tooth flank are reduced from 0.98 (tooth tip) and 2.01 (tooth flank) to 0.84 and 1.77 due to the influence of the increased infeed radius.

\section{Derivation of recommendations}

When manufacturing gears by full-forward extrusion from different metallic materials, the flow behavior directly affects the process result. Challenges arise from inflow formation, insufficient die filling, especially at the beginning of the process, and low achievable strain hardening during forming of materials with low yield stress. In addition, the yield stress of the material has an effect on the achievable gearing accuracy. For a given material and lubricant system, the tool geometry offers a promising control lever to influence the material flow during forming and improve the achievable component and process properties for a given material. In the context of this contribution, the targeted adaption of the cold forging process by modification of the shoulder angle, the flank angle, and the infeed radius was investigated.

Based on the gained knowledge, concrete recommendations for the cold forging of gears can be derived. In addition to the targeted improvement of component properties, however, undesirable effects must also be taken into account. Fig. 18 summarizes and evaluates the findings on the influence of the investigated tool-side measures on the achievable component and process properties, in order to support the decision making process.

\section{Summary and outlook}

Due to its high material and energy efficiency, cold extrusion of gears offers a promising alternative to conventional gear manufacturing. For medium to high requirements on geometric and mechanical properties, net-shape gears can be manufactured in a process suitable for serial production. With respect to the broad field of application, there is a wide range of gear materials with different levels of strength to be formed. The varying flow behavior of different metallic materials significantly affects the process result in gear extrusion. This investigation identified material-specific challenges and the underlying cause-effects during the full forward extrusion of gears. On that basis, suitable tool-sided measures for the targeted improvement of component and process properties were evaluated. For this purpose, a numerical process model was built with the simulation software simufact.forming 14.0.1 and validated during the investigation. This model is suitable to determine the resulting component and process properties and identify the underlying mechanisms of action. The developed tool-sided measures have a positive influence on the component and process properties. The findings can be used for the targeted adaptation of component properties and the production of ready-to-use gears from non-ferrous and light metals. In the following, the identified material-specific 
Fig. 18 Evaluation matrix of the influence of the yield stress and tool-sided measures on the component and process properties

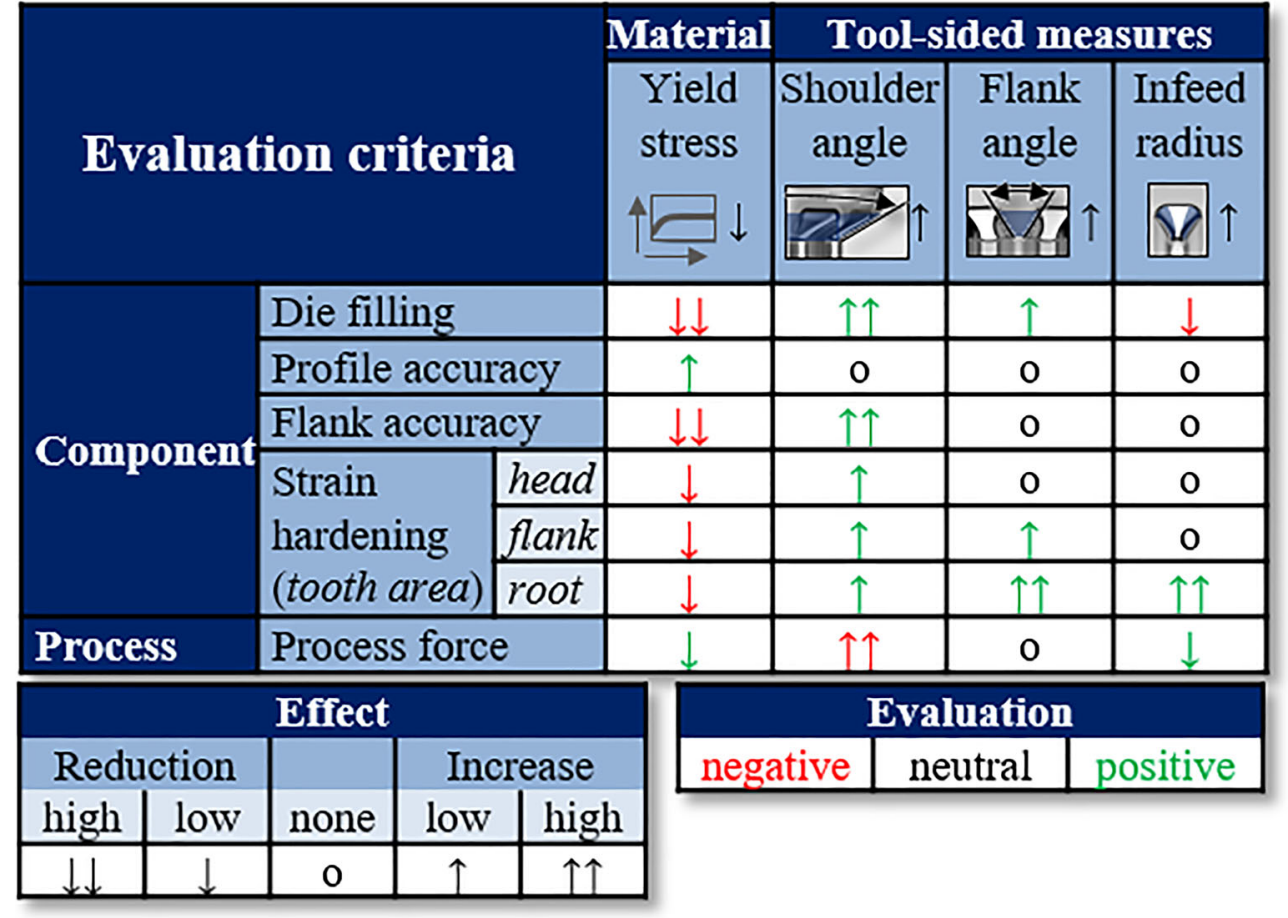

challenges and underlying cause-effects are summarized and the evaluated tool-sided measures are described:

\subsection{Material specific challenges}

- When forming materials with lower yield stress, locally higher material flow speeds and reduced tool contact occur at the beginning of the process due to the low flow resistance in the inflow area. This leads to insufficient die filling and inflow formation in the lower area of the gear as well as a reduced geometrical accuracy of the tooth flank (Fig. 9).

- Elevated forming forces due to a higher yield stress result in an increased elastic deformation and larger geometric deviation of the tooth profile (Fig. 10).

- With a lower yield stress of the gear material, the contact pressure and friction within the extrusion die are reduced. This results in lower work hardening of the tooth surface (Fig. 11).

\subsection{Tool-sided measures and cause-effects}

- Shoulder angle

Enlarged shoulder angle cause a stronger deflection and reduced material flow velocity. As a result, the strain hardening is increased $(+38 \%)$ and the overall die filling $(+3 \%)$ as well as the gearing accuracy are significantly improved (IT class 7 instead of 9).
- Flank angle

By attaching a flank angle, a tunnel is created and the deflection of the material towards the tooth root and flank area is increased, leading to improved overall die filling (+ $3 \%)$ and higher strain hardening $(+40 \%)$.

- Infeed radius

An adjusted inlet radius allows to locally adapt the material flow velocity in a targeted manner. This increases the resulting hardening in the application relevant tooth root area by $74 \%$.

With regard to future research, the numerical process model is qualified to further investigate the tool geometry as well as other influencing factors within process design. It should be examined whether interactions between the tool-sided measures exist and how they affect the process result. Furthermore, a detailed analysis of the tool load is of interest in order to evaluate the achievable lifetime of the adapted forming tools.

By conducting further experimental forming tests, the prognosis quality of the numerical process model should be verified beyond the forming tests, performed with the tool reference geometry within this investigation. This can be done by experimental realization of the presented tool-sided measures. Other potential influencing factors that should be investigated are the geometry of the formed gear and the tribological conditions during forming. The tooth geometry is defined in the gear design. Compared with the gearing investigated here, modified gear radii and helical or non-involute tooth profiles are possible. A change in the geometry to be produced has a direct effect on the material flow during the forming process. 
In addition to the tool and workpiece, the tribological conditions influence the resulting component and process properties during extrusion. Against this background, it should be investigated whether an adaptation of the tool surface can be used to influence the tribological conditions and the process result.

Authors' contributions Andreas Rohrmoser: data collection, analysis, original draft, editing.

Hinnerk Hagenah: project administration, supervision, reviewing, editing. Marion Merklein: project administration, supervision, reviewing, editing.

Funding Open Access funding enabled and organized by Projekt DEAL. The authors thank the German Research Foundation (DFG) for supporting the research project "FOR 2271 process oriented tolerance management based on virtual computer-aided engineering tools" under Grant number ME 2043/55-2.

Data availability The datasets generated and analyzed during the current study are available from the corresponding author on reason-able request.

\section{Compliance with ethical standards}

Conflict of interest The authors declare that they have no conflict of interest.

Ethical approval The research does not involve human participants or animals and the authors warrant that the paper fulfills the ethical standards of the journal.

Consent to participate It is confirmed that all the authors are aware and satisfied of the authorship order and correspondence of the paper.

Consent to publish All the authors are satisfied that the last revised version of the paper is published without any change.

\section{Code availability Not applicable.}

Open Access This article is licensed under a Creative Commons Attribution 4.0 International License, which permits use, sharing, adaptation, distribution and reproduction in any medium or format, as long as you give appropriate credit to the original author(s) and the source, provide a link to the Creative Commons licence, and indicate if changes were made. The images or other third party material in this article are included in the article's Creative Commons licence, unless indicated otherwise in a credit line to the material. If material is not included in the article's Creative Commons licence and your intended use is not permitted by statutory regulation or exceeds the permitted use, you will need to obtain permission directly from the copyright holder. To view a copy of this licence, visit http://creativecommons.org/licenses/by/4.0/.

\section{References}

1. Inc. GIA (2020) Gears, drives and speed changers - global market trajectory \& analytics, Dublin. https://www.asdreports.com/ market-research-report-471801/gears-drives-speed-changers

2. Davis JR (2005) Gear materials, properties, and manufacture. ASM International, Ohio
3. Gupta K, Laubscher R, Davim JP, Jain N (2016) Recent developments in sustainable manufacturing of gears: a review. J Clean Prod 112:3320-3330

4. Jeong MS, Lee SK, Yun JH, Sung JH, Kim DH, Lee S, Choi TH (2013) Green manufacturing process for helical pinion gear using cold extrusion process. Int J Precis Eng Manuf 14(6):1007-1011. https://doi.org/10.1007/s12541-013-0134-7

5. Weiß A, Deliktas T, Liewald M, Missal N (2020) Cold forging of gear components by a modified Samanta process. Forsch Ingenieurwes 84(3):215-221. https://doi.org/10.1007/s10010-020-00403-4

6. Jelaska D (2012) Gears and gear drives. John Wiley \& Sons Ltd, Hoboken

7. Niemann G, Winter H (1980) Machine elements: design and calculation in mechanical engineering, vol 2. Springer Verlag, Berlin

8. ICFG-Document (2002) Tool life \& tool quality in cold forging, part 1: general aspects of tool life. Doc. 14/02. Meisenbach Verlag Bamberg

9. Kiener C, Merklein M (2019) Researching of commonalities and differences in cold forging of spur and helical gears. Production Engineering 13(3-4):391-397. https://doi.org/10.1007/s11740019-00887-2

10. Behrens BA, Odening D (2009) Process and tool design for precision forging of geared components. Int J Mater Form 2(S1):125128. https://doi.org/10.1007/s12289-009-0577-7

11. Deutsches Kupferinstitut (2007) Kupfer-Zink-Legierungen (Messing und Sondermessing).

12. Hockett J, Sherby O (1975) Large strain deformation of polycrystalline metals at low homologous temperatures. J Mech Phys Solids 23(2):87-98

13. Schrader T, Shirgaokar M, Altan T (2007) A critical evaluation of the double cup extrusion test for selection of cold forging lubricants. J Mater Process Technol 189(1):36-44

14. Rohrmoser A, Kiener C, Hagenah H, Merklein M (2019) Influence of tribological conditions on application relevant component properties of cold forged gears. Prod Eng 13:579-588. https://doi.org/ 10.1007/s11740-019-00909-Z

15. Bay N (1994) The state of the art in cold forging lubrication. J Mater Process Technol 46(1-2): 19-40

16. DIN-Norm (1977) Modulreihe für Zahnräder - Moduln für Stirnräder. DIN 780-1. Beuth-Verlag, Berlin

17. Rohrmoser A, Heling B, Schleich B, Kiener C, Hagenah H, Wartzack S, Merklein M (2019) A methodology for the application of virtual evaluation methods within the design process of cold forged steel pinions. In: Proceedings of the 22th International Conference on Engineering Design (ICED19) pp. 3451-3460. https://doi.org/10.1017/dsi.2019.352

18. VDI-Standard (1986) Prestressed dies for cold forging. VDI 3176 Beuth-Verlag, Berlin

19. Volume A: theory and user information (2015) Marc $₫ 2015$.

20. ISO-Norm (2013) Cylindrical gears - ISO systems of accuracy - part 1: definitions and allowable values of deviations relevant to corresponding flanks of gear teeth ISO 1328-1. Beuth-Verlag, Berlin

21. Lange K, Kammerer M, Pöhlandt K, Schöck J (2008) Fließpressen: Wirtschaftliche Fertigung metallischer Präzisionswerkstücke. Springer Verlag, Berlin Heidelberg New York. https://doi.org/10. 1007/978-3-540-30910-9

22. Radzevich SP (2012) Dudley's handbook of practical gear design and manufacture, 2nd edn. CRC Press, Boca Raton

23. Bausch $\mathrm{T}$ (2015) Innovative Zahnradfertigung: Verfahren, Maschinen und Werkzeuge zur kostengünstigen Herstellung von Stirnrädern mit hoher Qualität, 5th edn. Expert Verlag, RenningenMalmsheim

Publisher's note Springer Nature remains neutral with regard to jurisdictional claims in published maps and institutional affiliations. 